\title{
How Salmonella Typhimurium measure the length of their Flagellar Filaments
}

\author{
J. P. Keener \\ Department of Mathematics \\ University of Utah
}

January 4, 2005

\begin{abstract}
We present a mathematical model for the growth and length regulation of the filament of the flagellar motor of Salmonella Typhimurium. Under the assumption that the molecular constituents are translocated into the nascent filament by an ATP-ase and then move by molecular diffusion to the growing end, we find a monotonically decreasing relationship between speed and velocity of growth that is inversely proportional to length for large length. This give qualitative but not quantitative agreement with data of th evelocity of growth.

We also propose that the length of filaments is "measured" by the rate of secretion of the $\sigma^{28}$-antifactor FlgM, using negative feedback, and present a mathematical model of this regulatory network.
\end{abstract}

Acknowledgment: This research was supported in part by NSF Grant DMS0211366.

\section{Introduction}

A central question in cell biology is how cells determine and regulate the size of their organelles. More generally, it is of interest to understand how physical and/or mechanical properties of a cell and its environment are measured [9].

The flagellar motor of Salmonella Typhimurium is an example of an organelle that is built to exacting standards. Morphologically, it is divided into three parts: the basal body, the hook, and the filament. The filament and hook are external to the cell, while the basal body is anchored in the inner and outer membranes. The filament is the largest portion of a flagellum, stretching to more than $10 \mu \mathrm{m}$, and when rotated by the motor at the base, serves as a propellor. The hook lies between the filament and the basal structure and works as a universal joint. A diagram of the flagellar complex is shown in Fig. 1.

The synthesis of the flagellar motor requires the expression of 50 genes, arranged in clusters of 17 operons, in a carefully controlled temporal order. These operons are divided into three classes according to their order of expression [7]. The master operon in the highest 


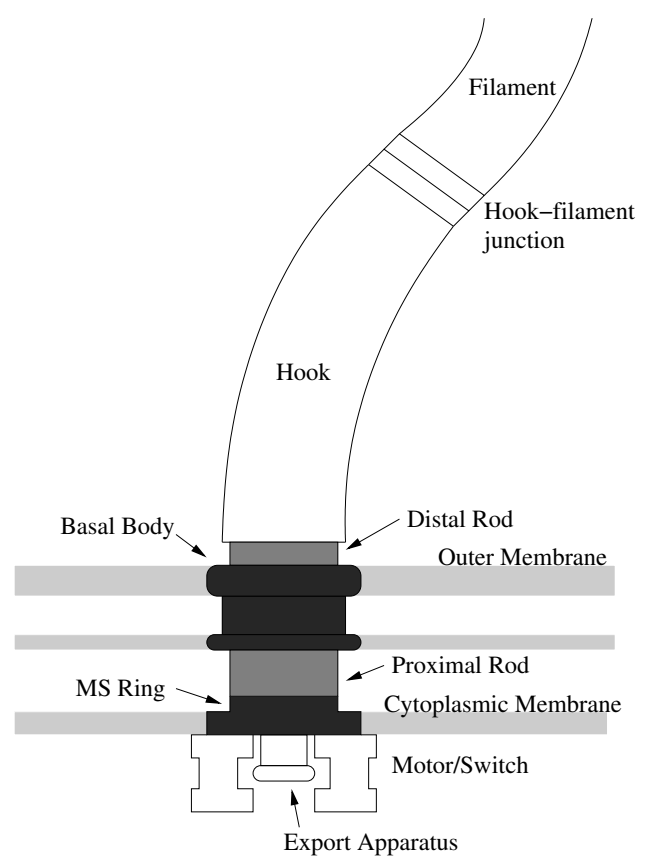

Figure 1: Schematic diagram of flagellar motor construction.

class governs the expression of the rest by activating operons in the second class. A flagellumspecific sigma factor in the second class regulates the expression of other operons in the same or lower classes. The basal structure and hook must be complete before the proteins for the filament are expressed, so that transcription of the filament components is "just-in-time" [11].

Assembly of the flagellum complex begins with the insertion of a ring structure within the cytoplasmic membrane. When this ring structure is completed, a secretion apparatus is constructed and inserted. The secretion apparatus is needed for export of flagellar structural subunits beyond the inner cytoplasmic membrane. Beyond the cytoplasm, secreted subunits self-assemble into the growing structure.

The nascent flagellum is a hollow cylindrical structure (outer diameter $20 \mathrm{~nm}$, inner diameter $2 \mathrm{~nm}$ ) that is formed when partially folded monomeric constituents reach the growing tip and polymerize. The monomeric subunits are secreted from the cellular cytoplasm following hydrolysis of ATP by the ATP-ase protein FliI. Secretion of the hook constituent protein, FlgE, proceeds until the hook is $55( \pm 6) \mathrm{nm}$ long. At that point, secretion switches to proteins FlgK and FlgL which form the hook-filament junction and then secretion of FliC, the filament constituent protein (flagellin), begins. A model for a possible mechanism by which the hook structure is measured and the secretion switch is made was presented in [6].

Filaments are typically $10 \mu \mathrm{m}$ long although the filament continues to grow indefinitely, while the velocity of growth decreases with length. Iino [5] measured the velocity of growth to be an exponentially decreasing function of length, with a typical velocity of growth for a filament in the range of $10-100 \mathrm{~nm} / \mathrm{min}$. Interestingly, if a filament is broken off, it will regrow with the same length dependent velocity as at first [5], indicating that the cell has some way of measuring the flagellar length and detecting the breakage.

The main questions that we wish to address in this paper are what determines the velocity 


\begin{tabular}{|l|l|}
\hline Protein & Function \\
\hline FliC & filament constituent protein \\
FliD & filament cap protein \\
FlgKL & hook-filament junction \\
FlgM & secretant/anti- $\sigma^{28}$ factor \\
$\sigma^{28}$ & RNA regulator \\
\hline
\end{tabular}

Table 1: Some proteins used in filament construction and their function.

of growth and how the bacterium measures and regulates the length of its filament. The short answer is that since movement of secretants through the tube is driven primarily by diffusion, the flux through the tube is ohmic and is therefore inversely proportional to the length of the tube. This length dependent flux is transduced into a chemical signal by means of a negative feedback circuit involving the secretant FlgM. Together, these enable the cell to measure filament length and respond to breakage. In the remainder of this paper we develop and analyze mathematical models of these processes.

\section{Length Dependent Growth Rate}

The first step in this analysis is to develop a model of length dependent secretion rate. An important fact is that the nascent tube has an inner diameter of about $2 \mathrm{~nm}$, which for steric reasons, means that movement of molecules must be in single file, with no passing permitted [8]. We suppose that the unfolded monomers are of length $l$.

We suppose that the secretion machinery is located at $x=0$ and that the growing tip is at $x=L(t)$. We let $p(x, t) d x$ be the probability that at time $t$ there is a molecular subunit whose trailing end lies between $x$ and $x+d x$ along the forming tube. Since molecules in the tube are of length $l$,

$$
\int_{0}^{L} p(x, t) d x=l N
$$

where $N$ is the number of monomers in the tube at time $t$. Standard conservation implies that

$$
\frac{\partial p}{\partial t}=-\frac{\partial J}{\partial x}
$$

where $J$ is the flux of probability.

If monomers move via a standard diffusion process, then $J=-D \frac{\partial p}{\partial x}$. It is worth noting that this is correct for a single file diffusion processes as long as we track the average flux of particles and not the movement of individual particles. It is useful to note that $\frac{J}{l}$ is the flux in units of numbers of molecules per time.

Very little is known about the diffusional movement of unfolded linear molecules in the forming tube. One thing that is clear is that the wall of the tube has a rapidly varying charge distribution that influences the movement of the molecules on a local spatial scale. This is similar to the effect that a charge distribution on the wall has on the movement of ions through an ion channel. It is possible to include the effect of these inhomogeneities in 
the description of the diffusion process, but because the effect is spatially periodic on a much smaller spatial scale than the length of the filament, it has little net effect on the average motion of molecules.

At the polymerizing end of the filament, there is a cap constructed of FliD. These proteins are thought to prevent the escape of $\mathrm{FliC}$ and to facilitate polymerization [4]. It follows that at the growing tip the flux of molecules must exactly match the rate of polymerization. Thus, at $x=L$,

$$
\frac{J}{l}=-\frac{D}{l} p_{x}=k_{p} p
$$

where $k_{p}$ is the rate constant for polymerization. In the Appendix, we derive a formula for $k_{p}$ from the folding energy of the monomer.

The secretion process is energized by an ATP-ase (FliI). It is not known exactly how this ATP-ase works, although there are hypothetical verbal models. One proposal [10] is that soluble FliI (complexed with FliJ) receives the export substrate from cytoplasmic chaperones, and delivers the substrate to a membrane associated structure, which then translocates it to the channel in the nascent structure.

To model this process, we assume that the membrane associated structure can be in one of two states, either empty awaiting binding with its cognate substrate, or bound and involved in translocation. It follows that the probability of going from the unbound to the bound state in time $\delta t$ is $K_{o n} \delta t=k_{o n} \frac{[S]}{K_{S}+[S]} \delta t$, where $[S]$ is the concentration of the substrate. Usually, we would assume that the probability of going from the bound to unbound state is $k_{o f f} \delta t$, however, because of the steric no-passing condition, translocation and unloading cannot take place unless there is open space in the filament for the subunit to move into. Thus, we assume that the probability of going from the bound to the unbound state is $k_{o f f}(1-p(0, t)) \delta t$. Thus, if $P$ is the probability that the membrane associated ATP-ase is bound by its cognate substrate $S$, then

$$
\frac{d P}{d t}=K_{o n}(1-P)-k_{o f f}(1-p(0, t)) P
$$

where $p(0, t)$ is the probability that the $x=0$ end of the nascent hook is occupied by a monomer at time $t$. The primary consequence of this statement is that translocation can be delayed if the forming hook is crowded with monomer. With these as the binding and unbinding rates, the flux of monomer at $x=0$ must be the same as the unbinding rate,

$$
\frac{J}{l}=k_{o f f}(1-p(0, t)) P .
$$

We take $P$ to be in quasi-steady state, so that

$$
P=\frac{K_{o n}}{k_{o f f}(1-p(0, t))+K_{o n}},
$$

implying that

$$
-\frac{D}{l} \frac{\partial p}{\partial x}=\frac{k_{o f f} K_{o n}(1-p(0, t))}{k_{o f f}(1-p(0, t))+K_{o n}} .
$$

at $x=0$. 
Finally, the length $L(t)$ of the growing filament is governed by the differential equation

$$
\frac{d L}{d t}=\frac{J}{\beta l}
$$

at $x=L$, where $\beta$ is the number of monomers per unit length of filament.

The equations (2)-(8) constitute a free boundary problem. To transform this into a problem on a fixed domain, we set $x=L(\tau) y, t=\frac{l^{2}}{D} \tau, s=\frac{L}{l}$, and from the chain rule learn that

$$
\frac{\partial}{\partial \tau}=\frac{l^{2}}{D} \frac{\partial}{\partial t}+L^{\prime}(\tau) y \frac{\partial}{\partial x}, \quad \frac{\partial}{\partial y}=L(\tau) \frac{\partial}{\partial x},
$$

so that

$$
\frac{\partial}{\partial x}=\frac{1}{L(\tau)} \frac{\partial}{\partial y}, \quad \frac{\partial}{\partial t}=\frac{D}{l^{2}} \frac{\partial}{\partial \tau}-\frac{D L^{\prime}(\tau) y}{l L(\tau)} \frac{\partial}{\partial y} .
$$

In these new coordinates, the partial differential equation (2) becomes

$$
\frac{\partial p}{\partial \tau}=\frac{s^{\prime}(\tau) y}{s(\tau)} \frac{\partial p}{\partial y}+\frac{1}{s^{2}} \frac{\partial^{2} p}{\partial y^{2}}
$$

where $s$ is the scaled filament length $s=\frac{L}{l}$, and the growth of the filament is determined by

$$
s^{\prime}=-\left.\frac{1}{\beta l s} p_{y}\right|_{y=1} .
$$

Now, set $u=s^{2}$ and find

$$
u \frac{\partial p}{\partial \tau}=\frac{y u^{\prime}}{2} \frac{\partial p}{\partial y}+\frac{\partial^{2} p}{\partial y^{2}}, \quad u^{\prime}=-\left.\epsilon p_{y}\right|_{y=1},
$$

where $\epsilon=2 \frac{1}{\beta l}$, with boundary conditions

$$
p_{y}=-\sqrt{u} \frac{l^{2}}{D} \frac{k_{o f f} K_{o n}(1-p(0, t))}{k_{o f f}(1-p(0, t))+K_{o n}} .
$$

at $y=0$ and

$$
p_{y}=-\sqrt{u} \frac{l^{2}}{D} k_{p} p,
$$

at $y=1$. Reasonable numbers for parameters are $\beta=2.4 / \mathrm{nm}$ and $l=75 \mathrm{~nm}$, so that $\epsilon=\frac{2}{\beta l}=0.0111$.

Numerical simulations show that $p$ quickly reaches quasi-steady state. This is because $u$, which acts as a time constant for the diffusion process, is slowly varying, so that the boundary conditions are slowly varying as well. This solution satisfies

$$
0=\frac{y u^{\prime}}{2} \frac{\partial p}{\partial y}+\frac{\partial^{2} p}{\partial y^{2}}, \quad u^{\prime}=-\left.\epsilon p_{y}\right|_{y=1} .
$$

Since $u^{\prime}$ is a scalar (as yet unknown), it must be that

$$
p_{y}=A \exp \left(-\frac{y^{2} u^{\prime}}{4}\right),
$$


and

$$
p(y)=A \int_{0}^{y} \exp \left(-\frac{y^{2} u^{\prime}}{4}\right) d y+B .
$$

It is useful to introduce the notation

$$
E\left(u^{\prime}\right)=\int_{0}^{1} \exp \left(-\frac{y^{2} u^{\prime}}{4}\right) d y
$$

so that

$$
p(0)=B, \quad p(1)=A E\left(u^{\prime}\right)+B, \quad p^{\prime}(0)=A, \quad p^{\prime}(1)=A \exp \left(-\frac{u^{\prime}}{4}\right),
$$

Then, the boundary conditions (14) and (15) become

$$
A=-s K_{D} \frac{(1-B)}{K_{a}(1-B)+1}
$$

and

$$
K_{b} A \exp \left(-\frac{u^{\prime}}{4}\right)=-s K_{D}\left(A E\left(u^{\prime}\right)+B\right),
$$

where $K_{D}=\frac{k_{o f f} l^{2}}{D}, K_{a}=\frac{k_{o f f}}{K_{o n}}$, and $K_{b}=\frac{k_{o f f}}{k_{p}}$. The consistency condition is

$$
u^{\prime}=-\epsilon A \exp \left(-\frac{u^{\prime}}{4}\right)
$$

and the flux is

$$
j=\frac{J}{l k_{o f f}}=\frac{1-B}{K_{a}(1-B)+1} .
$$

Eliminating $A$ and $B$ from these, we find

$$
s=\frac{1}{K_{D} j E\left(u^{\prime}\right)}\left(1-\frac{j}{1-K_{a j}}-K_{b} j \exp \left(-\frac{u^{\prime}}{4}\right)\right)
$$

and

$$
u^{\prime}=\epsilon \frac{\exp \left(-\frac{u^{\prime}}{4}\right)}{E\left(u^{\prime}\right)}\left(1-\frac{j}{1-K_{a j}}-K_{b} j \exp \left(-\frac{u^{\prime}}{4}\right)\right) .
$$

Since $s=\sqrt{u}$, the equations (25) and (26) can be viewed as an implicit differential equation for $u^{\prime}$ as a function of $u$, which is not easily interpreted. However, since $\epsilon$ is quite small, we can find a meaningful approximate equation

$$
\frac{d s}{d \tau}=\frac{1}{2} \epsilon K_{D} j, \quad s=\frac{1}{K_{D} j}\left(1-\frac{j}{1-K_{a} j}-K_{b} j\right),
$$

which is valid to leading order in $\epsilon$. When $K_{a} j<<1$ this reduces further to (in dimensional units)

$$
\frac{d L}{d t}=\frac{1}{\beta} \frac{k_{o f f}}{\frac{k_{o f f} L}{D}+1+\frac{k_{o f f}}{k_{p}}}
$$




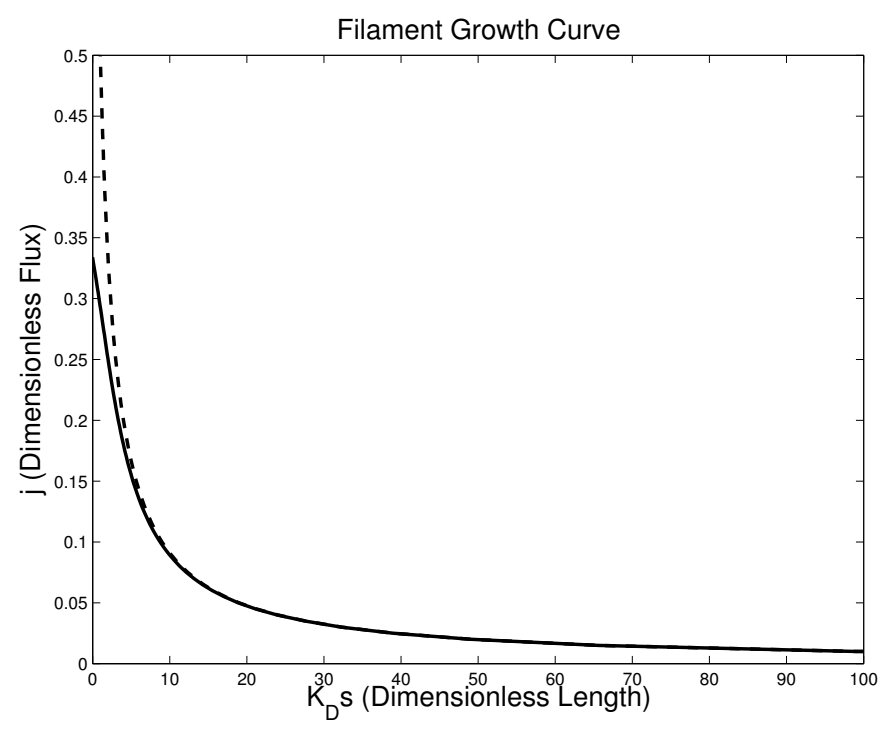

Figure 2: Flux $j$ (dimensionless) of monomer as a function of filament length with $K_{a}=2$ (solid curve), and with $K_{a}=0$ (dashed curve).

or even simpler as

$$
\frac{d L}{d t}=\frac{D}{\beta l} \frac{1}{L},
$$

for $L$ sufficiently large.

The main conclusion of this calculation is that there is a velocity-length relationship $\frac{d L}{d t}=V(L)$ which is a monotone decreasing function of $L$, and which for sufficiently large $L$ is inversely proportional to $L$. This velocity-length relationship is plotted in Fig. 1, for $K_{a}=2$ (solid curve) and $K_{a}=0$ (dashed curve), and $K_{b}=0$. Increasing $K_{b}$ merely shifts these curves to the left.

These velocity-length curves exhibit the correct qualitative behavior. The only available data [5] show velocity-length curves for filaments to be well fit by decaying exponentials for filaments in the range of $4-12 \mu \mathrm{m}$. Possible explanations for why there is not quantitative agreement with data is discussed below.

\section{Filament Length Control}

Filament length is not tightly regulated. However, if they are broken off, filaments regrow at a rate that is similar to that of the original growth [5], indicating that the length of the filament is somehow sensed by the bacterium.

The ability to sense the length of a filament is a consequence of the gene regulatory network that produces filament protein FliC (flagellin). Two important components of the filamental regulatory system are the transcription factor $\sigma^{28}$ and its inhibitor FlgM. $\sigma^{28}$ is a class 2 factor while FlgM is both class 2 and class 3 . Being class 2 factors, both $\sigma^{28}$ and FlgM are produced during the second stage of construction, while the hook is being constructed.

$\sigma^{28}$ is an activator of RNA polymerase that is necessary for transcription of all class 3 
factors, including the flagellar protein FliC. When possible, $\sigma^{28}$ binds with core RNA to form activated RNA polymerase, called $\mathrm{E} \sigma^{28}$, and transcription of class 3 factors proceeds. However, FlgM inhibits the activity of $\sigma^{28}[1]$. Thus, as long as there is an excess of FlgM, the activity of $\sigma^{28}$ is inhibited, and class 3 transcription is prevented.

Before the hook assembly is complete, the ATP-ase that drives secretion is selective for hook protein FlgE. Upon completion of the hook, the specificity of the ATP-ase is modified so that hook protein is no longer secreted and proteins necessary for the completion of the filament are secreted. At this time, FlgK, FlgL, and FlgM become substrates for the ATP-ase and so are secreted. FlgK and FlgL are class 2 factors that stabilize the hook structure and provide a junction between hook and filament. The secretion of FlgM, however, acts as a signal that flagellar construction is about to begin. As FlgM is secreted, $\sigma^{28}$ is released from its inactivation, and class 3 factors FlgM and FliC are produced. As long as FlgM is secreted, this production continues. However, if the secretion slows (as it will as the flagella grows longer), the concentration of FlgM begins to build up again, once again inhibiting $\sigma^{28}$ activity, shutting off the production of FliC. However, if the filament is broken off, secretion of FlgM increases dramatically (since the secretion rate is length dependent), setting off a signal that more FliC is needed for the reconstruction project.

We wish to develop a mathematical model of this regulatory process. The chemical reactions are as follows: It is known that $\sigma^{28}$ forms a complex $\mathrm{E} \sigma^{28}$ with core RNA, via

$$
\sigma^{28} \underset{k_{-\sigma}}{\stackrel{k_{\sigma}}{\rightleftarrows}} \mathrm{E} \sigma^{28} .
$$

It is suggested that FlgM inhibits the activity of $\sigma^{28}$ in three ways [1]. It binds with $\sigma^{28}$, it probably destabilizes $\mathrm{E} \sigma^{28}$, causing $\sigma^{28}$ to unbind from the core RNA, and it may bind to $\mathrm{E} \sigma^{28}$, preventing polymerase activity. Thus, we assume that FlgM forms a complex with $\sigma^{28}$

$$
\mathrm{FlgM}+\sigma^{28} \underset{k_{-m}}{\stackrel{k_{m}}{\rightleftarrows}} C_{\sigma} .
$$

We model the interaction of FlgM with $\mathrm{E} \sigma^{28}$ by assuming that they form a complex $C_{\sigma}^{*}$ via

$$
\mathrm{FlgM}+\mathrm{E} \sigma^{28} \underset{k_{-m^{*}}}{\stackrel{k_{m^{*}}}{\rightleftarrows}} C_{\sigma}^{*},
$$

and that $C_{\sigma}^{*}$ degrades to $C_{\sigma}$ via

$$
C_{\sigma} \stackrel{{ }^{k_{C}}}{\rightleftarrows} C_{\sigma-C}^{*} .
$$

Because this scheme forms a loop, the principle of detailed balance requires that

$$
\frac{k_{C}}{k_{-C}} \frac{k_{-m^{*}}}{k_{m^{*}}}=\frac{k_{-m}}{k_{m}} \frac{k_{\sigma}}{k_{-\sigma}}
$$

Finally, FlgM is produced at a rate proportional to $\sigma^{*}$ and secreted (after the hook is completed), while $\sigma^{28}$ is gradually degraded. 
The differential equations for the biochemistry are as follows. We denote $M=[\mathrm{FlgM}]$, $\sigma=\left[\sigma^{28}\right], \sigma^{*}=\left[\mathrm{E} \sigma^{28}\right], C=\left[C_{\sigma}\right]$, and $C^{*}=\left[C_{\sigma}^{*}\right]$. Then,

$$
\begin{aligned}
\frac{d M}{d t} & =k_{*} \sigma^{*}-\mu_{M}-k_{m} \sigma M+k_{-m} C-k_{m^{*}} \sigma^{*} M+k_{-m^{*}} C^{*} \\
\frac{d \sigma}{d t} & =-k_{m} \sigma M+k_{-m} C-k_{\sigma} \sigma+k_{-\sigma} \sigma^{*} \\
\frac{d \sigma^{*}}{d t} & =-k_{m^{*}} \sigma^{*} M+k_{-m^{*}} C^{*}+k_{\sigma} \sigma-k_{-\sigma} \sigma^{*} \\
\frac{d C}{d t} & =k_{m} \sigma M-k_{-m} C-k_{C} C+k_{-C} C^{*} \\
\frac{d C^{*}}{d t} & =k_{m^{*}} \sigma^{*} M-k_{-m^{*}} C^{*}+k_{C} C-k_{-C} C^{*}
\end{aligned}
$$

Here we have ignored the degradation of $\sigma^{28}$. Finally, we assume that the production of flagellar protein FliC is governed by

$$
\frac{d F}{d t}=\rho k_{*} \sigma^{*}-\mu_{F},
$$

where $F=[\mathrm{FliC}]$, assuming that $\rho$ copies of FliC are produced for every copy of FlgM. The rates of secretion are

$$
\mu_{F}=\frac{F}{F+M} \mu_{s e c}, \quad \mu_{M}=\frac{M}{F+M} \mu_{s e c}
$$

where $v \mu_{\text {sec }}=\frac{J}{l}$, and $v$ is the control volume in which the concentration of FlgM and FliC are measured. This assumes that molecules FlgM and FliC are secreted at a rate that is proportional to their relative concentrations, since both are secretion targets for the secretion machinery. The rate of secretion is determined by the diffusion model proposed in section 2 , namely, with the flux-length relationship given by (27), and $K_{\text {on }}$ related to substrate concentration through

$$
K_{\text {on }}=k_{\text {on }} \frac{[S]}{K_{S}+[S]}, \quad[S]=[F]+[M] .
$$

This relationship assumes that FlgM and FliC are the sole targets of secretion. In fact, FliD (cap protein) is also secreted during filament construction, but we ignore this secretion substrate here.

To simplify these equations somewhat, we assume that the biochemistry is in quasiequilibrium, in which case

$$
\sigma^{*}=\frac{\sigma_{0} K_{\sigma} K_{M}}{M+K_{M}}, \quad C+C^{*}=\frac{\sigma_{0} M}{M+K_{M}}
$$

where

$$
K_{M}=\frac{k_{\sigma}+k_{-\sigma}}{k_{\sigma} \frac{k_{m^{*}}}{k_{-m^{*}}}+k_{-\sigma} \frac{k_{m}}{k_{-m}}}, \quad K_{\sigma}=\frac{k_{-\sigma}}{k_{-\sigma}+k_{\sigma}}
$$


With this approximation, the equation for $M$ becomes

$$
\frac{d}{d t}\left(v M+\frac{v \sigma_{0} M}{M+K_{M}}\right)=K_{*} \frac{K_{M}}{M+K_{M}}-\frac{M}{S} \frac{J}{l} .
$$

Similarly, equation (40) becomes

$$
\frac{d(v F)}{d t}=\rho K_{*} \frac{K_{M}}{M+K_{M}}-\frac{F}{S} \frac{J}{l}
$$

where $K_{*}=k_{*} v \sigma_{0} K_{\sigma}$.

The flagellar length is slowly increasing, related to the rate of secretion of FliC,

$$
\frac{d L}{d t}=\frac{F}{S} \frac{J}{\beta l}
$$

at $x=L$. Since the relative amounts of FliC and FlgM secreted can vary, equation (47) should include the time delay $\tau=\frac{L}{J}$ to account for transport delay from the time a molecule enters the $x=0$ end until it arrives at the $x=L$ end, but we ignore this complication here. The relationship between $J$ and $L$ is given by

$$
\frac{L l}{D}=\frac{l}{J}-\frac{1}{k_{o f f}-\frac{1}{K_{o n}} \frac{J}{l}}
$$

Since this is an implicit relationship for $\frac{J}{l}$ as a function of $L$, it is useful to rewrite (47) as an equation for $\frac{J}{l}$ as

$$
\frac{d}{d t}\left(\frac{J}{l}\right)\left(l \frac{d L}{d J}\right)=\frac{F}{S} \frac{J}{\beta l}
$$

and use (48) to determine that

$$
l \frac{d L}{d J}=\frac{D}{l}\left(-\frac{l^{2}}{J^{2}}-\frac{1}{K_{o n}} \frac{1}{\left(k_{o f f}-\frac{1}{K_{o n}} \frac{J}{l}\right)^{2}}\right) .
$$

In this way, we have the system of three differential equations (45), (46), and (49) for $v M$, $v F$, and $\frac{J}{l}$ with $L$ determined from these through (48).

\subsection{Parameter Values}

The data for length-velocity relationships for filaments are sparse. Iino [5] found lengthvelocity curves for three strains of Salmonella that were well fit by curves of the form

$$
V=V_{0} \exp (-\kappa L)
$$

with $\kappa=0.27 / \mu \mathrm{m}$, and $V_{0}$ ranging between 0.25 and $0.55 \mu \mathrm{m} / \mathrm{min}$. However, $V_{0}$ is not the maximal velocity since this fit is valid only for filaments in the range of $4-14 \mu \mathrm{m}$. (A fourth strain of Salmonella had much slower velocities with $V_{0}=0.02 \mu \mathrm{m} / \mathrm{min}$.) Using this data, we estimate $\frac{l}{D}=1.3 \times 10^{-3} \mathrm{~min} / \mu \mathrm{m}$. 
Table 2: Table of filament parameter values

\begin{tabular}{|c|c|}
\hline$\beta$ & $2.4 / \mathrm{nm}$ \\
$\frac{l}{D}$ & $1.0 \times 10^{-3} \mathrm{~min} / \mu \mathrm{m}$ \\
$k_{\text {on }}$ & $2500 / \mathrm{min}$ \\
$k_{\text {off }}$ & $500 / \mathrm{min}$ \\
$\frac{1}{k_{p}}$ & 0 \\
$v \sigma_{0}$ & 0 \\
$K_{*}$ & $500 / \mathrm{min}$ \\
$v K_{S}$ & 10 \\
$v K_{M}$ & 10 \\
$\rho$ & 5 \\
\hline
\end{tabular}

It is known that $\frac{k_{-m}}{k_{m}}=200 \mathrm{pM}$, and $\frac{k_{-m^{*}}}{k_{m^{*}}}=800 \mathrm{pM}[2]$. Thus, it must be that

$$
\frac{k_{-C}}{k_{C}}=4 \frac{k_{-\sigma}}{k_{\sigma}} .
$$

This implies that the complex $C^{*}$ is less stable than $\mathrm{E} \sigma^{28}$, so that FlgM indeed destabilizes the complex $C^{*}$. Using these equilibrium constants, we find

$$
K_{M}=800 \frac{k_{\sigma}+k_{-\sigma}}{k_{\sigma}+4 k_{-\sigma}} \mathrm{pM}
$$

The remaining parameter values are unknown, but were estimated to give reasonable agreement with data, and are shown in Table 2.

\subsection{Results}

The qualitative behavior of these equations is readily deduced. Initially (at the termination of hook construction), $M$ is large, being a class 2 product, and $F=0$. With $M$ initially much larger than $K_{M}, \sigma^{28}$ is effectively inhibited and there is no production of class 3 factors. When the hook is completed, secretion specificity is switched so that secretion of $M$ begins. Subsequently, $M$ decreases to small levels, inducing the production of both $F$ and $M$. As $F$ increases, filament growth increases at first, but as the length of the filament increases, the rate of secretion drops, while both $M$ and $F$ continue to increase. This increase of $M$ causes the rate of production of both $F$ and $M$ to decrease, to match the decreased need for these.

If a filament is suddenly shortened, this growth scenario is repeated with a minor modification. Since the levels of both $F$ and $M$ are high when the shortening occurs, the initial growth rate is large as the stores of $F$ are used up. As $F$ and $M$ drop, however, their production rates are again increased, allowing continued regrowth.

Numerical simulation of these equations demonstrates this scenario. In Fig. 3 is shown the length as a function of time of a filament that starts at length $L=55 \mathrm{~nm}$, and grows until length $L=20 \mu \mathrm{m}$, at which point it is broken off to $L=2 \mu \mathrm{m}$ and then regrows. In Fig. 4 are shown the numbers of intracellular FlgM and FliC molecules during this process. 


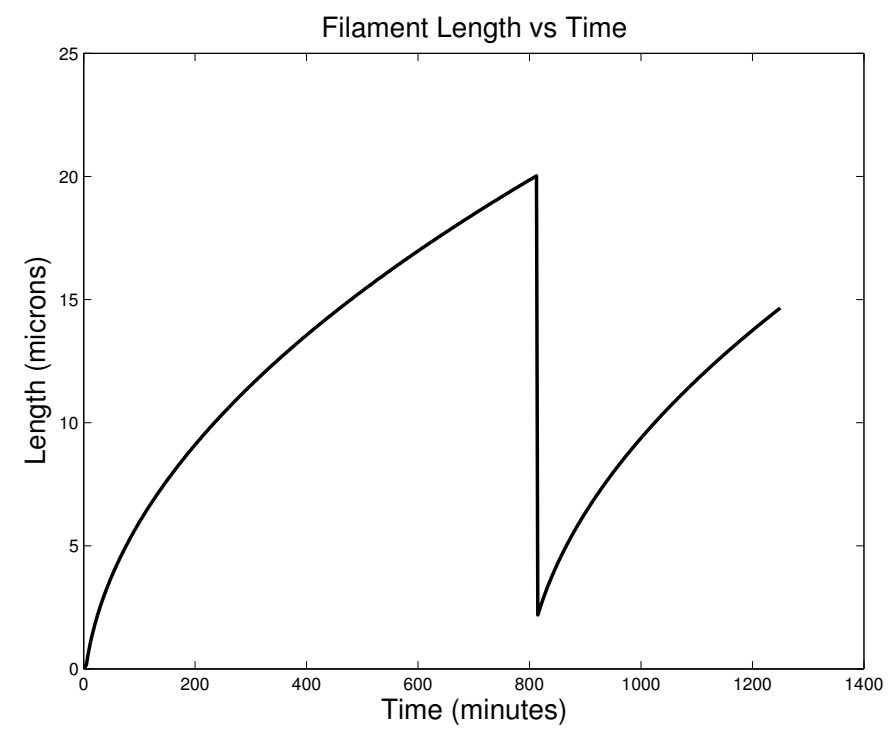

Figure 3: Length of a filament as a function of time.

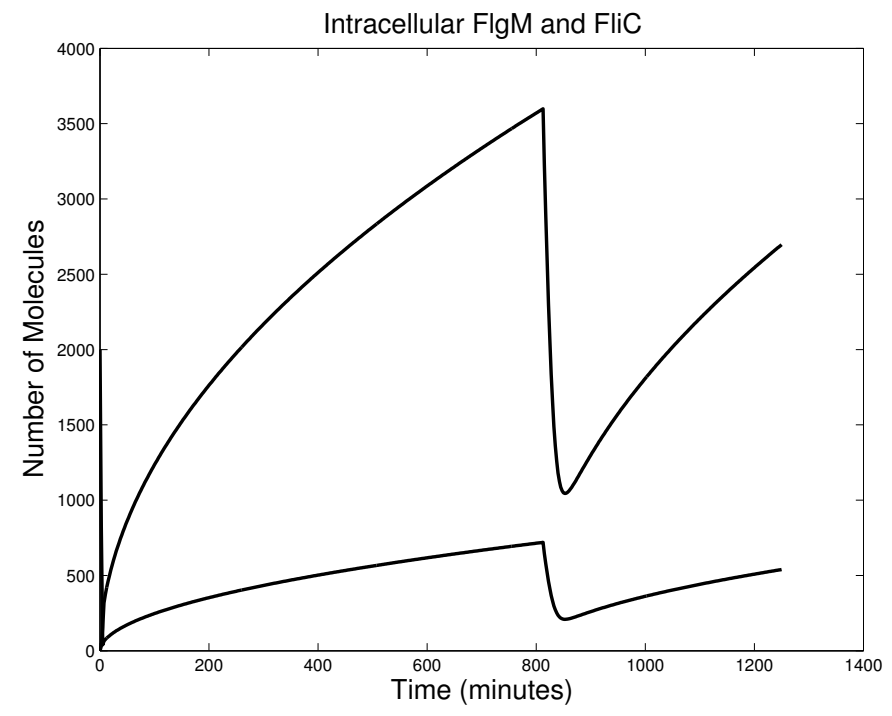

Figure 4: Quantities of intracellular FlgM (lower curve) and FliC (upper curve) during the growth of a filament. 


\section{Discussion}

Here we have presented a model based on physical mechanisms for the growth of flagellar filaments. We find a monotonically decreasing relationship between speed and velocity that is inversely proportional to length for large length. This agrees qualitatively but not quantitatively with measurements that find exponential decay, rather than algebraic. That velocity should be inversely proportional to length is not surprising. In fact, this is the expected result for any diffusive process.

The fact that our model finds algebraic decays whereas data show exponential decay is disturbing. There are two possible explanations for this discrepancy. First, it could be that the movement of monomers is not by a standard diffusive process. In a narrow channel, it is certain that monomer-wall interactions are significant and this will affect the details of the molecular movement. However, it is unlikely that this will change the overall behavior from an effective diffusion process.

It has been suggested (privately) that there should be a Boltzmann factor appearing somewhere. For example, if polymerization could be viewed as escape from a potential well, the depth of which was proportional to the length of the filament, then the mean escape time, and hence velocity of growth, would be exponential in length. However, a mechanistic explanation for this effect is not obvious.

The second possible explanation for this discrepancy lies with the interpretation of the data. The conclusion that the velocity is exponential is made using roughly a half dozen data points over one order of magnitude of lengths. Curiously, the data lie exactly on the fit curve, with no error. It is difficult to believe that the experimental procedures (especially when measuring a velocity, which involves finite differences) were as accurate as this suggests.

The model presented here finds that length detection is accomplished by the combination of a length dependent flux of a secreted molecule with a negative feedback on the production of the molecule.

A similar mechanism is used for quorum sensing in P. aeruginosa [3]. With quorum sensing, there is positive feedback for the production of the diffusible molecule. Here, when the diffusible molecule moves freely to the exterior of the cell, its concentration remains at low levels within the cell. However, when outward diffusion is slowed, because of high levels of the molecule in the extracellular space, intracellular levels of the molecule build up, triggering an autocatalytic switch which upregulates this and other products. In P. aeruginosa, the diffusible molecule is called an auto-inducer.

\section{Appendix}

Here we derive the boundary condition 3). It is clear that it is energetically favorable for flagellin molecules to be folded rather than unfolded. Thus, the polymerization process is fueled by the energy of folding. We can model this by assuming that near the growing end of the tube, molecules experience a force so that for $L-l<x<L$,

$$
J=-\frac{W^{\prime}(x)}{\nu} p-D p_{x}
$$


where $W(x)$ is the potential energy well associated with folding, and $\nu$ is molecular viscosity. We expect that $W^{\prime}(x)<0$ so that folding has the effect of pulling the molecule out of the tube. Furthermore, $p=0$ for $x \geq L$. Since $l / L$ is small, the diffusion process should be in quasi-steady state in the small (boundary layer) region $L-l<x<L$. Thus,

$$
p(x)=\frac{J}{D} \int_{x}^{L} \exp \left(\frac{W(\eta)-W(x)}{\nu D}\right) d \eta
$$

on the interval $L-l<x<L$. For $x<L-l, J=-D p_{x}$, so to match the flux $J$, it must be that

$$
p(L-l)=-p_{x}(L-l) \int_{L-l}^{L} \exp \left(\frac{W(\eta)-W(L-l)}{\nu D}\right) d \eta
$$

which is of the form

$$
\frac{D}{l} p_{x}(L-l)=-k_{p} p(L-l)
$$

with

$$
k_{p}=\frac{D}{l}\left(\int_{L-l}^{L} \exp \left(\frac{W(\eta)-W(L-l)}{\nu D}\right) d \eta\right)^{-1} .
$$

In the simple case that $W^{\prime}(x)=-F$ is a constant, we find that

$$
k_{p}=\frac{\Delta W}{l^{2} \nu}\left(1-\exp \left(-\frac{\Delta W}{D \nu}\right)\right)^{-1} .
$$

where $F l=\Delta W$ is the depth of the potential energy well.

\section{References}

[1] M. S. Chadsey, J. E. Karlinsey, and K. T. Hughes. The flagellar anti-sigma factor FlgM actively dissociates Salmonella typhimurium $\sigma^{28}$ RNA polymerase holoenzyme. Genes and Development, 12:3123-3136, 1998.

[2] G. S. Chilcott and K. T. Hughes. Coupling of flagellar gene expression to flagellar assembly in salmonella enterica serovar typhimurium and escheria coli. Microbiology and Molecular Biology Reviews, 64:2000, 2000.

[3] J. D. Dockery and J. P. Keener. A mathematical model for quorum sensing in pseudomonas aeruginosa. Bull. Math, Biol., 63:95-116, 2001.

[4] K. T. Hughes and P. D. Aldridge. Putting a lid on it. Nature Structural Biology, 8:96-97, 2001.

[5] T. Iino. Assembly of salmonella flagellin in vitro and in vivo. J. Supramolecular Structure, 2:372-384, 1974.

[6] J. P. Keener. A model for length control of flagellar hooks of salmonella typhimurium. J. Theoretical Biology, 2005. Accepted November, 2004. 
[7] K. Kutsukake, Y. Ohya, and T. Iino. Transcriptional analysis of the flagellar regulon of salmonella typhimurium. J. Bacteriol., 172:741-747, 1990.

[8] R. M. Macnab. How bacteria assemble flagella. Annual Review of Microbiology, 57:77$100,2003$.

[9] W. F. Marshall. Cellular length control systems. Annual Review of Cell and Developmental Biology, 20:677-693, 2004.

[10] T. Minamino and R. M. Macnab. Interactions among components of the salmonella flagellar export apparatus and its substrates. Molecular Microbiology, 19:1-5, 2000.

[11] A. Zaslaver, A. E. Mayo, R. Rosenberg, P. Bashkiin, H. Sberro, M. Tsalyuk, M. G. Surette, and U. Alon. Just-in-time transcription program in metabolic pathways. Nature Genetics, 36(5):486, 2004. 